\title{
Capturing and Quantifying Prototype Progress in a Design Course Using Automated Means
}

\author{
Sohail Ahmed Soomroa,b*, Yazan Barhousha, Iván Sánchez Milaraa, Zhengya Gonga, Georgi V. \\ Georgieva \\ a Center for Ubiquitous Computing, University of Oulu, Finland

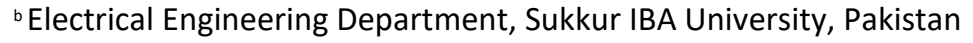 \\ *Corresponding author e-mail: sohail.soomro@oulu.fi
}

\begin{abstract}
:
This study aimed to capture the prototyping process in the context of design education using automated means. We used a course on creative design to deploy and test a system comprising blog-based documentation and a prototype capturing photo booth. Over the time span of several weeks, we gathered data relative to students' weekly exercises and prototyping of an individual project. We discuss the data gathered and quantified from the course in terms of weekly patterns, daily patterns, general prototyping, and documentation characteristics as well as delve deeper into three example projects. The results demonstrate that this system can help students document and organise their prototypes at various stages. As the system provides automatic and effortless documentation, it can motivate students to document while designing. It can also be used to track the progress of students and permit instructors to take corrective action when a student is not progressing as expected.
\end{abstract}

Keywords: prototyping; digital fabrication; documentation, design process

\section{Introduction}

Ldjkfld Iterative prototyping is central to design education (Wensveen \& Matthews, 2014). However, it is challenging to capture and quantify the phases in the process of prototyping (Camburn et al., 2017; Sjöman et al., 2017). This lack of structured design process documentation makes it difficult to evaluate related research findings (Zimmerman et al., 2010). Reflective design documentation (Dalsgaard \& Halskov, 2012; Ghajargar \& Wiberg, 2018), on the other hand, allows designers to understand how artefacts evolve during the design phase, which can help them understand the design process.

In the case of prototypes in particular, previous studies have addressed the importance of digitally capturing physical prototypes during the early stages of product development (PD) (Gibson et al., 2004; Kohtala et al., 2018; Soomro et al., 2021). Dedicated tools have been developed to capture early-stage prototypes through multi-view images and associated metadata, such as the name of the designer, location, and time (Erichsen et al., 2020; Sjöman et al., 2017). The main motivation behind 
these tools is to digitally capture the development process to aid understanding of prototyping in the early stages of PD projects.

It is important to note that documentation and reflection activities, along with prototyping, are essential in the context of design education (Agouridas \& Race, 2007; Milara et al., 2019). In this study, we examined how to make documentation and reflection tasks easier for students and instructors to allow them to dedicate more time to idea generation, prototyping and iterations.

Although built-in progress documentation systems that promote process-like documentation are currently available (e.g. Bracewell et al., 2009; Tseng, 2015), they do not support the dedicated capture of prototypes. We aimed to address this gap in our study. In particular, we envisioned a system that automates the documentation of physical artefacts (i.e. prototypes) in terms of imagery, along with the corresponding descriptions and reflections in the context of design education. Our focus was to include both the ideas generated in the design process and the snapshots through the prototyping process. We believe that this will contribute to the devising of future aids and interventions to support design education.

We introduced a Protobooth Oulu v.2, a physical artefact capturing device, to facilitate documentation for the purpose of supporting design education. The primary goal of our proposed system was to help design education students reflect on their concepts by reviewing, describing and reflecting on their prototypes. A key feature of Protobooth is that prototypes are captured as output from the design activity, thereby ultimately facilitating research into the activity. The link between the activity and the artefact (prototype) is a core concept and assumption of that work (Sjöman et al., 2017; Soomro et al., 2021; Yang, 2005).

\section{Related work}

\subsection{Capturing prototype images with Protobooth}

Sjöman et al. (2017) presented the Protobooth system to help researchers obtain more data during the course of projects. The system is used to capture physical prototypes from ongoing early-stage product development (PD) projects with the aim of giving researchers access to information and knowledge from fuzzy front-end and early-stage PD.

Kohtala et al. (2018) discussed various ways of representing a prototype repository, including possible accessibility through virtual reality. This system produces a basis for documentation and feedback, helps explore potentially supportive methods (e.g. photogrammetry) in the early stages of $P D$, and highlights the limitations of capturing design output from projects.

Erichsen et al. (2019) used Protobooth to capture multi-view images together with metadata indicating by whom, when and where the prototypes were captured. This work demonstrated that such a capturing system could gather empirical data for enriching product development case studies on early-stage projects that involved prototyping for concept generation.

Although previous implementations of Protobooth Oulu exist (Barhoush et al., 2019), the current implementation offered the possibility for students to define the stage of the prototype (e.g. initial, middle, final) and the level of satisfaction of the student with the artefact being presented.

\subsection{Current system compared to the previous iteration}

This second iteration of the Protobooth system is designed to be deployed in educational environments. Its overall aim is to support the prototyping process of developing products during courses by facilitating student documentation and providing instructors with temporal data on the prototyping output. While this current iteration has its roots in the Protobooth system (Sjöman et al., 2017), it explicitly targets documentation in educational environments by integrating the image capturing system with documentation and simple feedback prompts. 
Compared to the previous implementation of the Protobooth Oulu system, we included, in part, the design rationale at the time of capture in the form of simple evaluation of stage of prototype and satisfaction level. This inclusion addressed the nonvisual aspects of prototype documentation-the purpose and success of the prototype. See Figure 1 for a comparison of the two system.

Protobooth Oulu v.1

\begin{tabular}{l|l}
\hline $\begin{array}{l}\text { Durability } \\
\begin{array}{l}\text { Portability } \\
\text { Social }\end{array}\end{array}$ & $\begin{array}{l}\text { Less durable material and not reusable } \\
\text { (needs to be manufactured again) } \\
\text { More clunky (Feels as if it is falling apart) }\end{array}$ \\
$\begin{array}{l}\text { User Interface } \\
\text { Blog posts are available for viewers in a timely } \\
\text { manner. }\end{array}$ \\
$\begin{array}{l}\text { RFID unique card ID (UID) is given to each user. } \\
\text { There is no account associated with the card (card } \\
\text { ID is parsed into the post title when created) }\end{array}$ \\
$\begin{array}{l}\text { Functionality } \\
\text { Ease of setup and } \\
\text { maintenance }\end{array}$ & $\begin{array}{l}\text { Limited to making the posts using the Protobooth } \\
\text { itself. Users could not modify or add to the posts } \\
\text { after posting } \\
\text { Requires writing a lot of code instead using out- } \\
\text { of-box packages. It cannot be fixed remotely. } \\
\text { Much slower (images are captured each solely). }\end{array}$
\end{tabular}

Protobooth Oulu v.2

Extremely durable material, ready for placement and use.

Solid build but has a heavier structure.

Blog posts can be categorized and tagged in any manner desired.

A customizable touch display interface with all the usernames. Each user has an account on the blog and usernames on the display are directly associated with the blog accounts.

Utilizes WordPress: users could make blogposts through their user accounts and could modify these posts (or posts captured using the Protobooth) at any point.

Backed up and ready for deployment when needed. It can be monitored and fixed remotely.

Simultaneous captures, where adding more cameras would not affect the speed

Figure 1. Comparison of the two versions of the Protobooth systems

\subsection{Aims}

We aimed to capture and quantify the prototyping and documentation process in the context of design education using automated means. To address this aim, we used a course on creative design to deploy and test a system comprising a blog-based documentation system and prototype capturing photo booth-the Protobooth Oulu v.2.

\section{Method}

This research consisted of three parts. The first part was the creative design course in which the experiment was performed. A WordPress-based blog was used for content management. The Protobooth Oulu v. 2 was used to capture information from prototypes and store it on a blog.

\subsection{Creative Design Course}

The MSc course (equivalent to 5 ECTS, or approximately 135 study hours) introduces creative problem-solving, design thinking and systematic ideation approaches, in teams and as individual work, by employing low-resolution prototyping and relying on the FabLab tools and processes. The course has a strong focus on horizontal competencies and interdisciplinarity, particularly on creativity and critical thinking. The course is assessed based on weekly tasks and a final project in which students need to build a prototype of an interactive artefact that somehow addresses human emotion, e.g. evoking surprise. The final project requires only the building of a low-fidelity prototype (Erichsen et al., 2020) for demonstration purposes. The course duration is seven weeks, and only students from education programs run by the (anonymised) can join. Typically, 30 to 40 students take part in the course annually, divided into exercise groups of a maximum of 20 people.

\subsection{Blog}

We used WordPress 5.2.4 as a content management system, hosted in the cloud, for course announcements as well as documentation of students' weekly assignments and the final project. We created a single blog in the system to document the work of all course students. All students were 
added to the system as users and were given permission to author either public or private blog posts. Private posts could be seen only by their authors and the course staff. We implemented this system to protect the privacy of the students, enabling them to decide if they would like to share their project tasks with the rest of the course participants.

When writing a blog post, for weekly assignments, students were instructed to create or choose tags (Figure 2). Tags are one of the pre-defined taxonomies in WordPress, which users can add to their WordPress blog posts to classify them into specific topics. A Secured shell (SSH) command-line interface for WordPress (WP-CLI) was installed on a web server alongside WordPress to allow for the updating of content and configuring of site installations without using a web browser, which was useful for the Protobooth implementation.

\subsection{Protobooth Oulu v.2}

Protobooth Oulu v. 2 was the automated photo capturing and publication system that sent captures to the aforementioned blog. The blog post was created only after the students took a picture. Next, students were able to edit the content using the WordPress web user interface (UI). It is important to note that students were required to add a description of their work after they used the Protobooth.

The outer container of Protobooth v. 2 is a CNC-cut wooden box (Figure 3 ). The interior is covered with sheets of white polycarbonate plastic to improve picture quality. Three camera modules were placed at different angles to capture the images. Each camera was attached to a Raspberry Pi 3 B+ microcontroller. A 3.5" touch screen display was attached to a fourth microcontroller to register user input. A multiple-window graphical user interface (GUI) was installed on the microcontroller with the touch screen display (Figure 4).

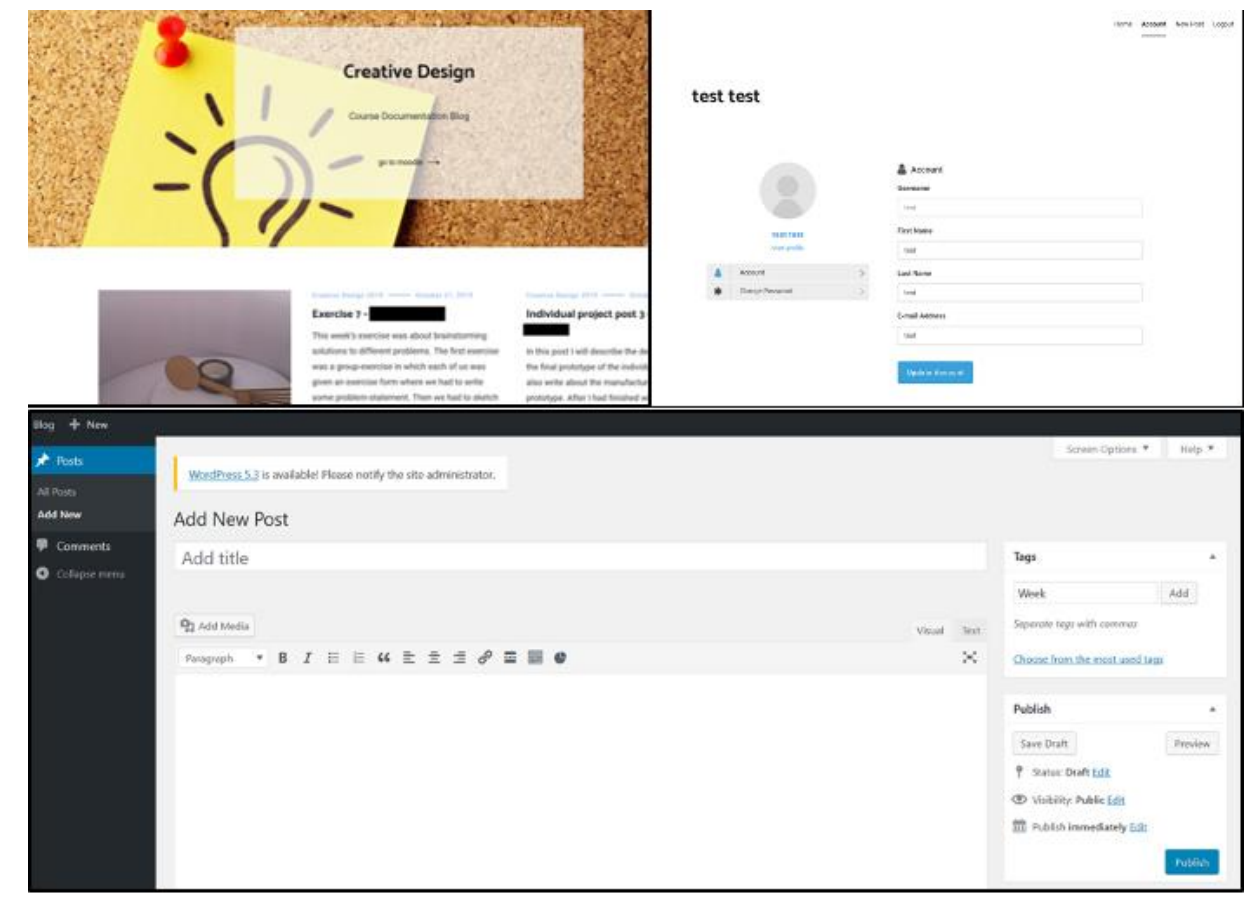

Figure 2. Interface of the system: Home page (upper left), User account (upper right) and the Interface for publishing a new post with Tags (bottom). 

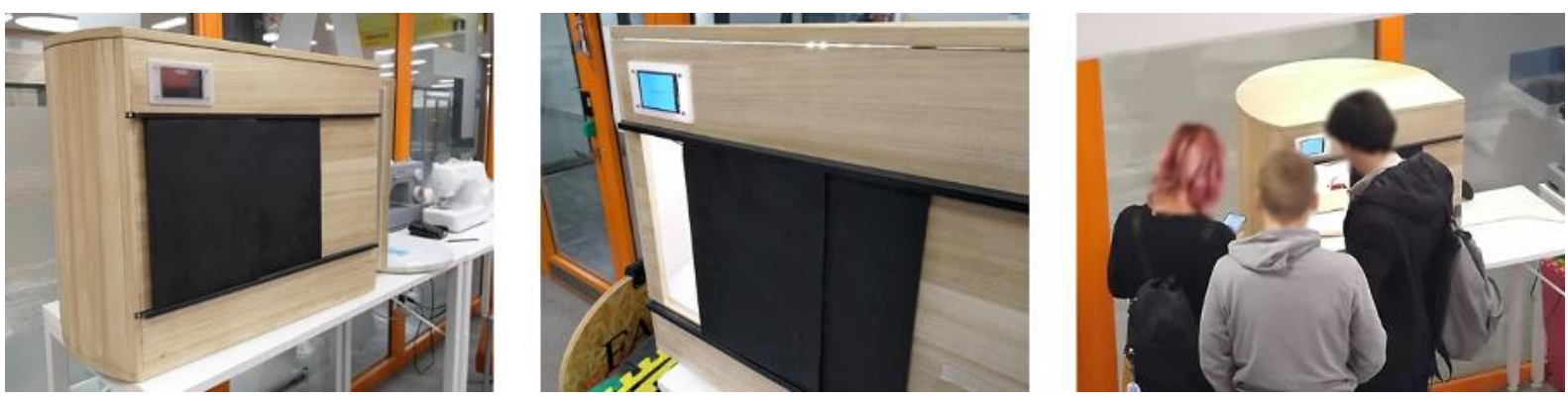

Figure 3. Protobooth Oulu v.2 in use. Students documenting a prototype
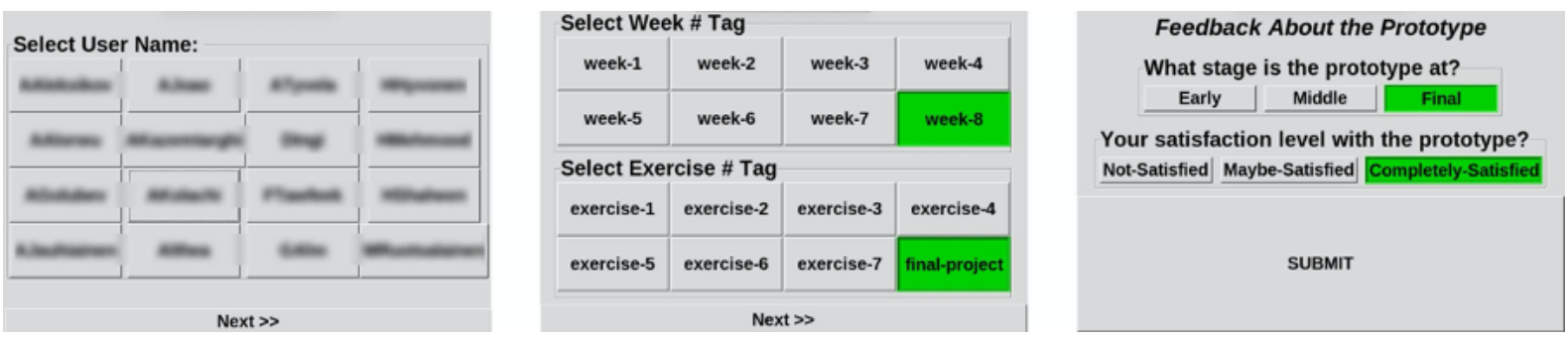

Figure 4. GUI of the system: user selection (left), course week/exercise selection (middle) and feedback on stage of prototyping and satisfaction level (right).

To make an automated blog post using Protobooth Oulu v.2, students selected their blog user name in the first window, added tags in the second and submitted their feedback (prototype stage and satisfaction level) in the third. Tags were used in the blog to identify the exercise or project and to register the feedback from the Protobooth. Students could create their own tags. A publish-subscribe network protocol transported a message with the input to each microcontroller with a camera module. Finally, the three microcontrollers with camera modules simultaneously captured an image and posted it to the blog using the WP-CLI. The GUI would then reset to accept a new input. The process, from input submission to GUI reset, took fewer than ten seconds.

The overall documentation of the prototyping process included images, displayed as blog posts, that allowed users to edit these blog posts by adding documentation in the edited post or in a separate blog post.

\section{Results}

\subsection{Posts and captures}

The course included seven exercises, completed either in teams of various sizes or individually, and one project consisting of an idea individually generated and materialised. We asked students to document their work, putting particular emphasis on reflection on the work they had done.

A total of 37 students used the blog and Protobooth Oulu v. 2 in the class. They published 605 posts over the course of 79 days.

\subsection{Prototyping stages and satisfaction levels}

The setup of Protobooth Oulu v.2 included students' own feedback on the following criteria ( also see Figure 4, right):

- Stage of prototyping (early, middle or final).

- Satisfaction level (not satisfied, maybe satisfied or completely satisfied).

It should be noted that, for every capture, the feedback on the two criteria was optional. 
Figure 5 shows the stages of prototyping as indicated during the captures. The early-stage prototyping label was used in 11 out of 30 student cases. When both middle-stage and final-stage labels were used, there were often more final-stage labels than middle-stage.

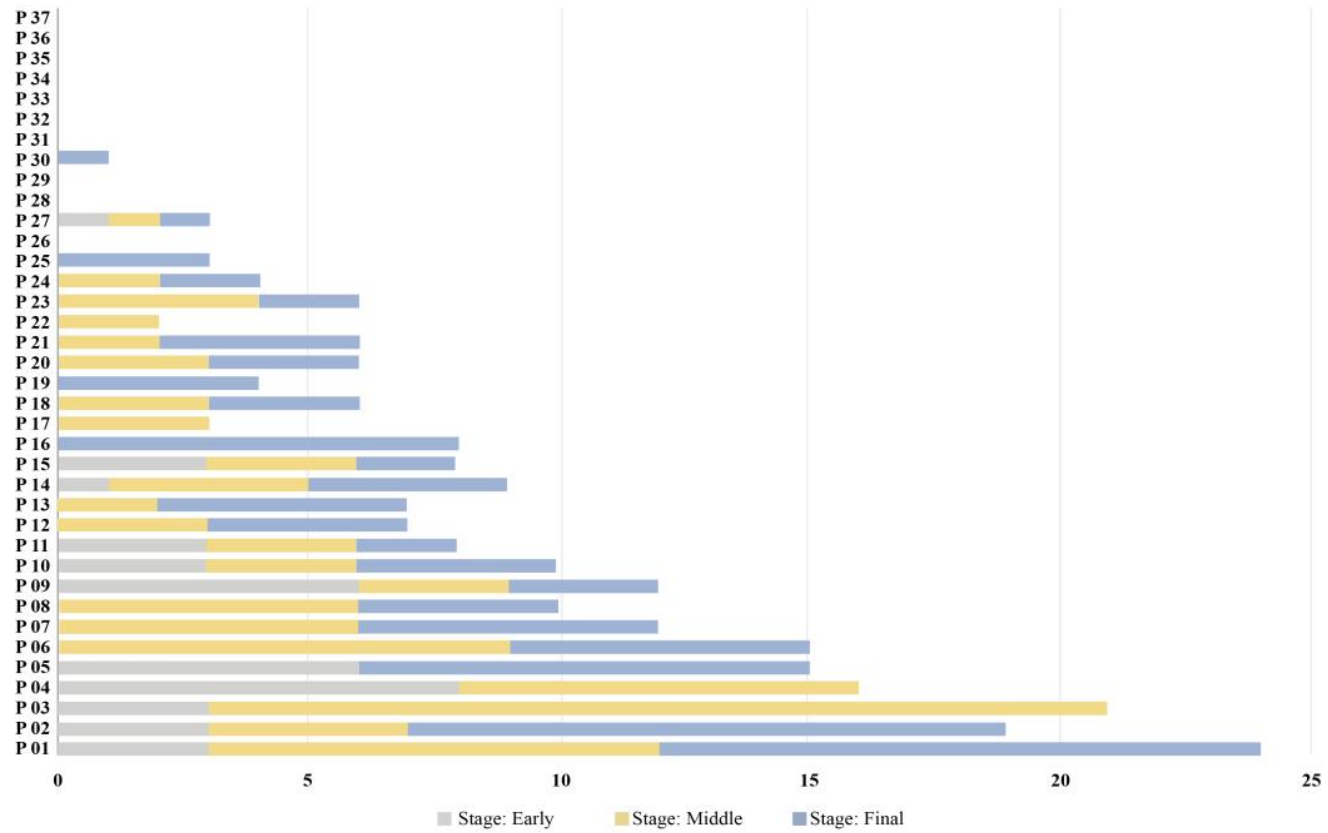

Figure 5. Student-indicated prototyping stages per individual project prototype capture (early, middle or final) accumulated according to user. Users are indicated with the letter $P$ and a number. Users without captures used other means to document their prototypes.

Figure 6 shows the students' satisfaction levels as indicated during the captures. Notable is that only four students used the 'not satisfied' label. The 'completely satisfied' label was used less often than 'maybe satisfied'. Nine students used only the 'maybe satisfied' label in their captures.

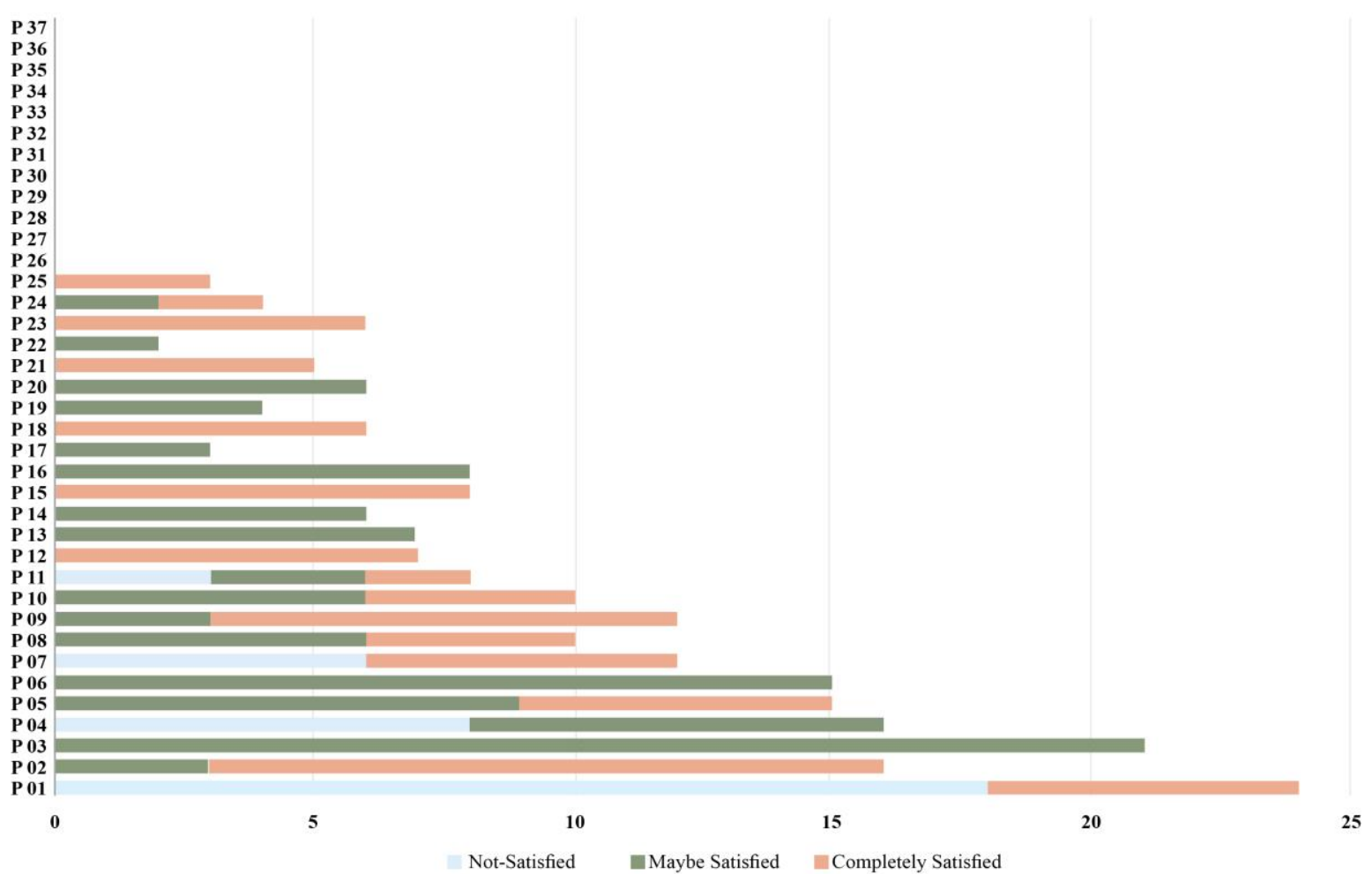

Figure 6. Student-indicated satisfaction levels per individual project prototype capture (not satisfied, maybe satisfied or completely satisfied) accumulated according to user. Users are indicated with the letter $P$ and a number. Users without captures used other means to document their prototypes. 


\subsection{Further analysis of three cases}

To delve deeper into the captured data, we selected three different cases as examples for further analysis (denoted by P02, P05 and P28, Figure 7). P02 used the Protobooth system for prototype captures, P05 used the Protobooth system and a mobile phone camera for prototype captures, and P28 used a mobile phone camera for prototype captures. All three provide examples of documentation that cover the seven exercises of the course and the individual project.

Figure 8 shows the posts according to the day of the week they were posted, including seven exercises and individual projects. The number of posts was higher in the first three days, which might have been because the exercises sessions were scheduled on these days. The lesser number of posts on weekends could have been due to having limited access to the Protobooth during weekends. We can also see in Figure 8 that P02 and P05 were active in terms of posts as compared to P28. Figure 9 shows the time of day of the posts of the three selected cases.

Figure 10 shows the length of the documentation blog posts of the three students. Short posts of up to 70 words were prevalent. There were also examples of very long posts of more than 1,000 words. Student P28 had less variety in terms of length of documentation posts.

Looking back to the data of P02 and P05 in Figures 5 and 6 (P28 used a different means of capturing), captures of the final stage are prevalent. P02 used the 'completely satisfied' label much more than the 'maybe satisfied' label, whilst P05 used 'maybe satisfied' more often than 'completely satisfied'.


Figure 7. Three example projects: Piano lamp (P02 top, a piano that lights up a lamp and a wall switch that plays the piano); Webby, the Suspicious Baby Sitter (P05 middle, a chair that will change when it is in use. This change, as convenient it might be, is also somewhat alarming and questionable); and a 3D puzzle that gives the false illusion that it can be solved (P28 bottom). 


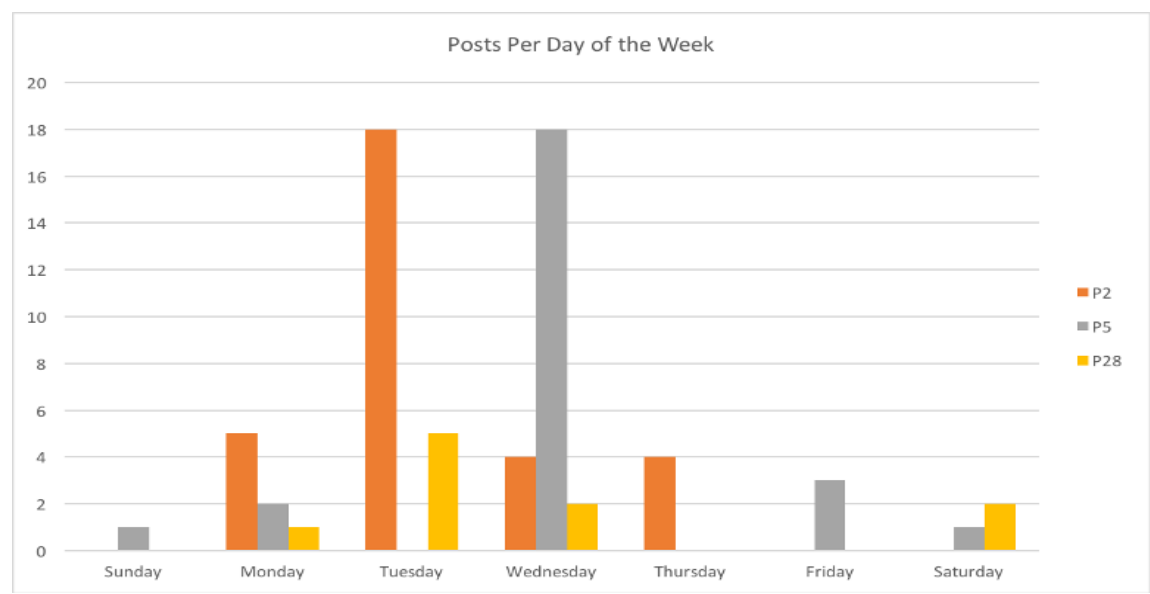

Figure 8. Documentation blog posts of the three selected cases according to the day of the week they were posted.

Posts Per Hour

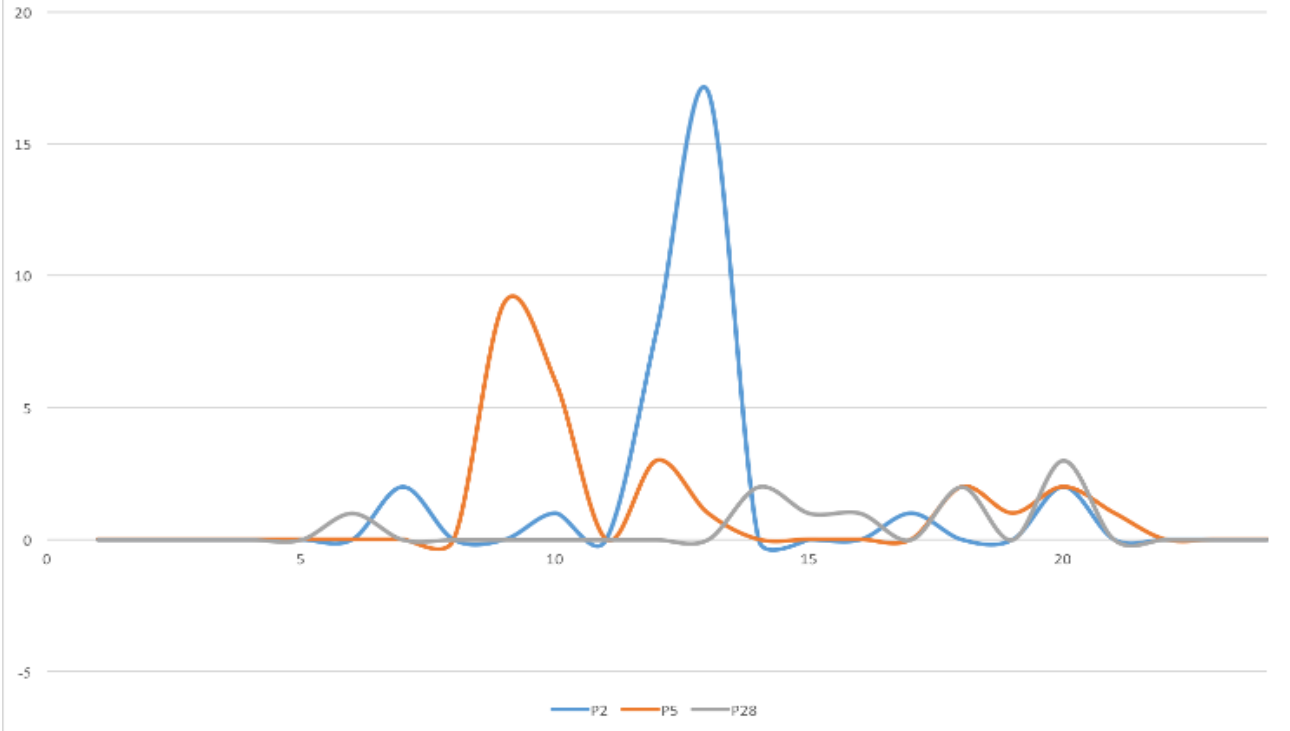

Figure 9. Time of day the documentation blog posts were posted by the three selected cases.

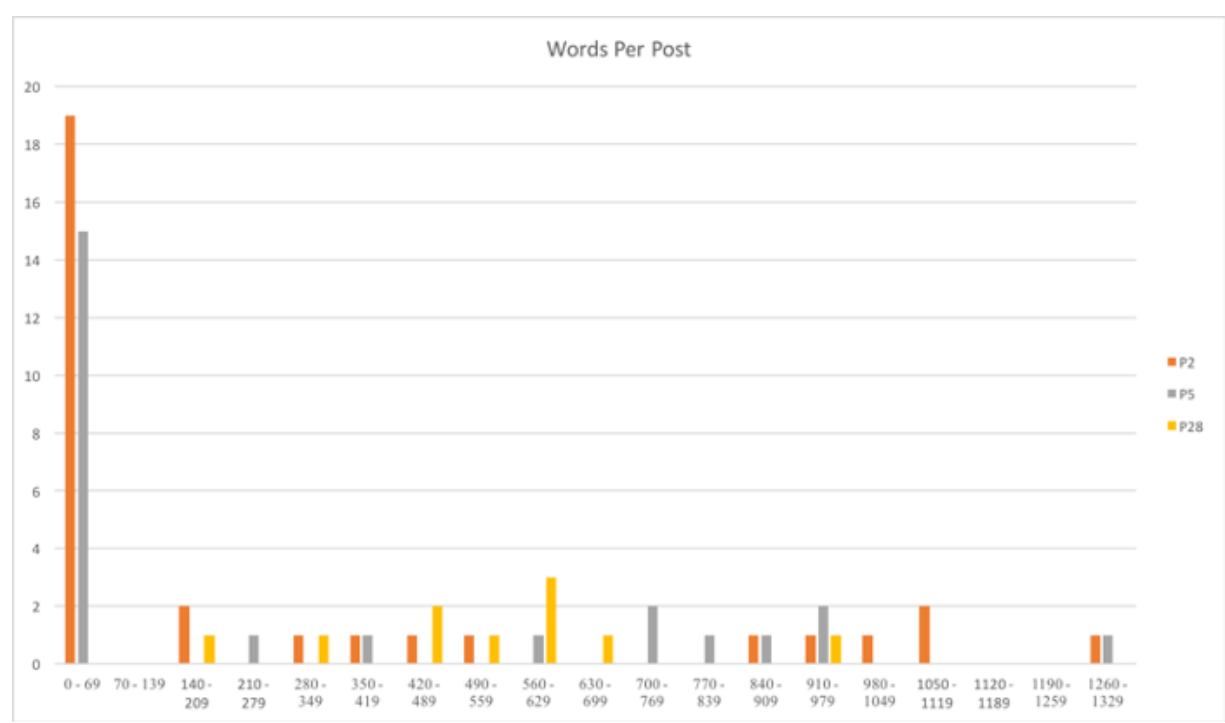

Figure 10. Length of the documentation blog posts of the three selected cases. 


\section{Discussion}

\subsection{Interpretation of the captured data and its utility in design education}

These cases can be quantitatively interpreted. For example, P02 had multiple captures of the final stage of the project. Many of the captures were labelled as 'satisfied', and there were many small posts and several very long posts.

When capturing prototypes over time, qualitative analysis of the captured data can convey the evolution of and major changes in the prototypes. We believe that such data is useful when trying to understand the process of prototyping and provides a means of improving prototypes and overall creative performance.

Qualitative interpretations can be made in terms of individual work. Frequency of captures and types of feedback might be indicative of an intensive prototyping process in terms of iterations and evolution. The time-related quantifiers (time of day, day of the week) might be dependent on individual schedules and work styles. Length of documentation, along with its frequency, might indicate an intensive prototyping process and strong reflection practices.

As the data were available throughout the progression of the course, we used these data to track students and assess their work. In particular, regularity and evolution of prototyping captures (either by Protobooth or by other means) were considered indicative of high performance by the student.

For example, unclear reflections in the documentation of some of the early exercises indicated that the reflection might be not sufficiently understood. In turn, this could prompt instructors to return to the topic of reflection. Furthermore, delayed documentation of some of the exercises might indicate that students could have been experiencing difficulty or finding these exercises less engaging, thus requiring reconsideration in future content delivery. The new features of the Protobooth helped us in the tracking and assessment phase. Further iterations of Protobooth Oulu will focus on new features that could help us apply interventions.

The results have implications for design education and the support of active interactions between instructors and students, particularly in larger design courses. The approach opens possibilities for real-time data analytics on the educational design process.

\subsection{Limitations of the current case}

Several limitations can be identified in relation to the current application case (i.e. course and system implementation). First, not all data were captured through Protobooth Oulu v.2, and we cannot quantify with the same precision the data captured through other means. Second, the feedback was limited in terms of options, including lack of an opt-out. We intend to address these limitations in future application cases in a different course and with improved system implementation. The system can be further improved in terms of the speed of capture, connectivity and functionality.

\section{Conclusion}

To capture and quantify the prototyping process in the context of design education with automated means, we used a course on creative design to deploy and test a system comprising a blog-based documentation system and prototype capturing photo booth. The gathered data of students' documentation and reflections on weekly exercises and prototyping of an individual project allowed us to interpret the results in terms of weekly patterns, daily patterns and general prototyping and documentation characteristics. The three example projects we examined in detail allowed us to gain insights in terms of individual work and intensity of the prototyping process, as well as indicators of the reflection practices of the students. Given the ease of use of the system, the students adopted it and used it for documentation throughout the course. 
The main contribution of this study is that the proposed system and feedback can be used to promptly track the progress of students and allow instructors to intervene when progress is not as expected. Moreover, the system is suitable for application in distributed and remote working situations. In future versions of the Protobooth system, we intend to implement design rationale with much more detail and test it in a different scenario.

\section{References}

Agouridas, V., \& Race, P. (2007). Enhancing knowledge management in design education through systematic reflection practice. Concurrent Engineering, 15(1), 63-76. https://doi.org/10.1177/1063293X07076267

Barhoush, Y. A., Erichsen, J. F., Sjöman, H., Georgiev, G. V., \& Steinert, M. (2019). Capturing prototype progress in digital fabrication education. Proceedings of the Design Society: International Conference on Engineering Design, 1(1), 469-478.

Bracewell, R., Wallace, K., Moss, M., \& Knott, D. (2009). Capturing design rationale. Computer-Aided Design, 41(3)(3), 173-186. https://doi.org/10.1016/j.cad.2008.10.005

Camburn, B., Viswanathan, V., Linsey, J., Anderson, D., Jensen, D., Crawford, R., Otto, K., \& Wood, K. (2017). Design prototyping methods: State of the art in strategies, techniques, and guidelines. Design Science, 3. https://doi.org/10.1017/dsj.2017.10

Dalsgaard, P., \& Halskov, K. (2012). Reflective design documentation. Proceedings of the Designing Interactive Systems Conference, 428-437. https://doi.org/10.1145/2317956.2318020

Erichsen, J. F., Sjöman, H., Steinert, M., \& Welo, T. (2020). Digitally capturing physical prototypes during early-stage engineering design projects for initial analysis of project output and progression. ArXiv:1905.01950 [Cs]. http://arxiv.org/abs/1905.01950

Ghajargar, M., \& Wiberg, M. (2018). Thinking with interactive artifacts: Reflection as a concept in design outcomes. Design Issues, 34(2), 48-63. https://doi.org/10.1162/DESI_a_00485

Gibson, I., Gao, Z., \& Campbell, I. (2004). A comparative study of virtual prototyping and physical prototyping. International Journal of Manufacturing Technology and Management, 6(6), 503-522. https://doi.org/10.1504/IJMTM.2004.005931

Kohtala, S. M. I., Erichsen, J. A. B., Sjöman, H., \& Steinert, M. (2018, August 14). Augmenting physical prototype activities in early-stage product development. DS 91: Proceedings of NordDesign 2018.

Milara, I. S., Georgiev, G. V., Riekki, J., Ylioja, J., \& Pyykkonen, M. (2017). Human and technological dimensions of making in fablab. Design Journal, 20, S1080-S1092. https://doi.org/10.1080/14606925.2017.1353052

Milara, I. S., Georgiev, G. V., Ylioja, J., Özüduru, O., \& Riekki, J. (2019). “Document-while-doing”: A documentation tool for Fab Lab environments. The Design Journal, 22(sup1), 2019-2030. https://doi.org/10.1080/14606925.2019.1594926

Sjöman, H., Erichsen, J. A. B., Welo, T., \& Steinert, M. (2017). Effortless capture of design output a prerequisite for building a design repository with quantified design output. 2017 International Conference on Engineering, Technology and Innovation (ICE/ITMC), 564-570. https://doi.org/10.1109/ICE.2017.8279935

Soomro, S. A., Barhoush, Y. A. M., Gong, Z., Kostakos, P., \& Georgiev, G. V. (2021). Tools for recording prototyping activities and quantifying corresponding documentation in the early stages of product development. Proceedings of the Design Society, 1, 3159-3168. https://doi.org/10.1017/pds.2021.577

Soomro, S. A., Casakin, H., \& Georgiev, G. V. (2021). Sustainable design and prototyping using digital fabrication tools for education. Sustainability, 13(3), 1196.

Tseng, T. (2015). Build in progress: building process-oriented documentation (pp. 237-254). https://doi.org/10.4324/9781315726519-16 
Wensveen, S., \& Matthews, B. (2014). Prototypes and prototyping in design research. In Prototypes and prototyping in design research (pp. 262-276). Routledge. https://doi.org/10.4324/9781315758466-25

Yang, M. C. (2005). A study of prototypes, design activity, and design outcome. Design Studies, 26(6), 649-669. https://doi.org/10.1016/j.destud.2005.04.005

Zimmerman, J., Stolterman, E., \& Forlizzi, J. (2010). An analysis and critique of research through design: Towards a formalization of a research approach. Proceedings of the 8th ACM Conference on Designing Interactive Systems, 310-319. https://doi.org/10.1145/1858171.1858228

Author Bios:

Sohail Ahmed Soomro is a Ph.D. researcher at the Center for Ubiquitous Computing, University of Oulu, Finland. His research interests are in digital fabrication, engineering design and user interactions in makerspace.

Yazan Barhoush is currently employed as a doctoral researcher in the Center for Ubiquitous Computing at the University of Oulu and as an xR \& Graphics developer at Nokia. His work is focused on digital fabrication, virtual reality, and $\mathrm{HCl}$.

Iván Sánchez Milara is a PhD student and teacher at University of Oulu, Finland, and instructor at FabLab Oulu. His research focus in integrating digital fabrication into formal education. His research interests also include $\mathrm{HCl}$, digital fabrication and learning technologies.

Zhengya Gong is a Ph.D. researcher at the Center for Ubiquitous Computing, University of Oulu, Finland. Her work focuses on design creativity, creative methods, design cognition, design thinking, and the effects of cultural bias in applying creativity methods.

Georgi Georgiev is an Associate Professor at the Center for Ubiquitous Computing, University of Oulu, Finland. His research interests are design creativity, idea generation, design cognition, digital fabrication and prototyping, design thinking, user interaction, and user experience.

Acknowledgements: This study has been partially financially supported by Academy of Finland 6Genesis Flagship (grant 318927), by EDUFI Fellowship (grant TM-20-11342), by China Scholarship Council (NO: 202107960006), and by the Erasmus+ project "Bridging the creativity gap" (agreement number 2020-1-UK-01-KA202-079124). 